\title{
Lipid changes during the perioperative period in patients with early breast cancer: a real-world retrospective analysis
}

Tao He ${ }^{1}$, Zhu Wang ${ }^{2}$, Yunhao $\mathrm{Wu}^{3}$, Xinyi Zhang ${ }^{3}, \mathrm{Xu} \mathrm{Li}^{4}$, Jiayuan $\mathrm{Li}^{5}$, Liang Du${ }^{6}$, Jie Chen ${ }^{7^{*}}$ and Qing Lv ${ }^{7}$

\begin{abstract}
Background: Surgery remains the major treatment for early breast cancer (BC), but surgery itself is also a trauma which might induce alterations in lipid metabolism. The aim of this study was to investigate the changes in lipid profiles and to explore factors associated with lipid changes pre- and postoperation.

Methods: We retrospectively analyzed the pre- and postoperative serum lipid profiles of 1934 BC patients.

Results: The levels of triglycerides (TG) $(p<0.001)$ and low-density lipoprotein cholesterol (LDL) $(p<0.001)$ were significantly elevated after surgery, while the levels of high-density lipoprotein cholesterol (HDL) $(p<0.001)$ were significantly decreased. After surgery, $27.76 \%$ of patients with preoperative ortholiposis developed dyslipidemia. Postmenopausal BC patients had a higher incidence of dyslipidemia (32.31\%) after surgery than premenopausal BC patients (26.07\%; $p=0.041)$. Additionally, patients with $\mathrm{BMI}>24(34.92 \%)$ had a higher incidence of dyslipidemia than patients with $\mathrm{BMI} \leq 24$ (24.84\%; $p=0.001)$. Moreover, the magnitudes of the TG increase $(p<0.001)$, cholesterol (TC) increase $(p=0.013)$ and LDL increase $(p=0.015)$ in the premenopausal group were all greater than those in the postmenopausal group. After adjusting for multiple baseline covariates, preoperative hyperlipidemia and progesterone receptor (PR)-positive status were significantly associated with elevated TG, TC and LDL levels after surgery.

Conclusions: Serum lipid profiles of BC patients may increase after surgery, especially premenopausal patients. Additionally, postmenopausal and overweight patients may have a higher risk of being diagnosed with dyslipidemia after surgery. Therefore, lipid monitoring, dyslipidemia prevention and corresponding interventions should be taken into consideration during the perioperative period.
\end{abstract}

Keywords: Breast cancer, Surgery, Dyslipidemia

\section{Introduction}

Breast cancer is one of the most prevalent malignancies worldwide [1]. According to the estimates from the Global Cancer Statistics, there were 2,088,849 newly diagnosed breast cancer cases in 2018, accounting for $24.2 \%$ of cancer cases among females [2]. In terms of mortality, breast cancer, accounting for $15 \%$ of cancer

\footnotetext{
*Correspondence: chenjiewestchina@163.com

${ }^{7}$ Department of Breast Surgery, West China Hospital, Sichuan University,

Chengdu 610041, Sichuan, China

Full list of author information is available at the end of the article
}

deaths among females, is the leading cause of female cancer-related deaths [3].

Currently, complete surgery remains the major curative treatment for localized breast cancer [4]. However, surgery itself is also a trauma that might induce the stress response of patients to surgery [5]. There are several systemic responses caused by surgical trauma, including endocrinological, immunological, hematological and psychological effects [6]. Recent studies have shown that carbohydrate, protein, fat, water and electrolyte metabolism might all be affected by the stress response to surgery [ 5 , 
7]. Among these changes, fat metabolism and serum lipid profile variations are one of the most important and obvious indicators.

Recently, some studies have revealed that dyslipidemia is associated with metastasis in patients with BC [7], and the regular oral administration of statins attenuates the spread of breast cancer metastases [8]. Additionally, dyslipidemia was an independent risk factor for inducing cardiovascular disease (CVD) [9]. A scientific statement from the American Heart Association revealed that CVD and breast cancer have several overlapping risk factors. Specifically, for older women, CVD poses a greater mortality threat than breast cancer itself [10]. As such, it is vital to monitor the serum lipid profiles of patients with $\mathrm{BC}$ after surgery in the clinic.

However, few studies have explored the serum lipid variations associated with the surgical stress response until now. Thus, we retrospectively investigated the serum lipid levels pre- and postoperation and explored the influencing factors of serum lipid changes.

\section{Methods and materials Patient selection}

The medical records of all consecutive patients diagnosed with early breast cancer were retrospectively collected from the Department of Breast Surgery, West China Hospital, Sichuan University, between February 2009 and December 2016. The Institutional Review Board and Ethics Committee of West China Hospital approved this retrospective study (IRB No. 2016L03115). Data were accessed anonymously, waiving the requirement for consent from study participants. All methods were carried out in accordance with the relevant guidelines and regulations. The inclusion criteria were as follows: (1) female patients aged $\geq 18$ years; (2) patients surgically treated and pathologically diagnosed with breast cancer; and (3) patients who had adequate organ function with Eastern Cooperative Oncology Group $(\mathrm{ECOG}) \leq 2$. The exclusion criteria were as follows: (1) patients with $\mathrm{BC}$ and other malignancies; (2) patients with distant metastatic breast cancer; (3) patients who previously underwent other operations; (4) patients who received neoadjuvant treatment; (5) patients taking drugs affecting lipid levels; (6) pregnant and lactating women; and (7) patients with incomplete data.

\section{Data collection and evaluated parameters}

Clinicopathological data were reviewed from medical records. The variables of interest included height, weight, body mass index (BMI), age at diagnosis, menopausal status, smoking status, tumor location, surgical procedures, Tumor Node Metastasis (TNM) stage [11], ER status, PR status, preoperative hypertension, preoperative hyperlipidemia and pre- and postoperative lipid profile values (blood samples were collected in the morning on an empty stomach within a week before surgery and within 2 weeks after surgery). According to the Chinese guidelines for the management of dyslipidemia, dyslipidemia was considered if patients met at least one of the following criteria: TG $\geq 1.7 \mathrm{mmol} / \mathrm{L}, \mathrm{TC} \geq 5.2 \mathrm{mmol} / \mathrm{L}$, $\mathrm{LDL} \geq 3.4 \mathrm{mmol} / \mathrm{L}$, and $\mathrm{HDL} \leq 1.0 \mathrm{mmol} / \mathrm{L}$ [12]. Body weight and height were assessed for the determination of BMI, which was calculated as the weight divided by the height squared $\left(\mathrm{kg} / \mathrm{m}^{2}\right)$. Patients were classified as postmenopausal if they were not pregnant, were aged over 40 years and had no menstruation for at least 12 months [13]. Since menopausal status is closely related to serum lipids, the participants in this study were further stratified into two groups by menopausal status: premenopausal and postmenopausal. Additionally, BMI may influence serum lipids [14], so we then stratified the participants into two groups: $\mathrm{BMI} \leq 24$ and $\mathrm{BMI}>24$.

\section{Statistical analysis}

All statistical analyses were performed using the Statistical Package for the Social Sciences (SPSS), version 22.0 for Windows (SPSS Inc., Chicago, IL, USA). Quantitative results are expressed as the mean \pm standard deviation (SD). A two-sided $p$-value of less than 0.05 was considered statistically significant. A paired-sample $\mathrm{T}$ test was used to compare the lipid values pre- and postoperation. One-way analysis of variance was used to assess significant differences in the magnitude of variations in lipid profiles pre- and postoperation between the premenopausal and postmenopausal groups and between the $\mathrm{BMI} \leq 24$ and $\mathrm{BMI}>24$ groups. The magnitudes of variation in lipid profiles pre- and postoperation were calculated, and the difference was calculated as the postoperative lipid values minus the preoperative lipid values. The Chi-square test was used to calculate the incidence of postoperative hyperlipidemia in patients with normal serum lipids before surgery. Moreover, we incorporated the significant variables associated with the increase in serum lipid profiles after surgery, which were identified by univariate analysis $(p<0.05)$, into a logistic regression model to identify the independent factors that were correlated with the elevated serum lipid profiles.

\section{Results}

\section{Patient characteristics}

A total of 1934 eligible and consecutive patients with $\mathrm{BC}$ were identified for inclusion in this retrospective cohort study. The mean age of the entire cohort was $48.90 \pm 5.38$ years, with a median age of 48 years old. The baseline characteristics of the patients were summarized in Table 1. Before surgery, all patients were 
Table 1 Demographic and clinical characteristics of the study population ( $n=1934)$

\begin{tabular}{|c|c|c|c|}
\hline & Premenopause $(\mathrm{N}=1184)$ & Postmenopause $(\mathrm{N}=750)$ & $p$ \\
\hline Age, mean (SD), y & $43.22(5.65)$ & $57.86(6.73)$ & $p<0.01$ \\
\hline $\mathrm{BMI}\left(\mathrm{kg} / \mathrm{m}^{2}\right), \mathrm{n}$ & & & $p<0.01$ \\
\hline$\leq 24$ & 824 & 441 & \\
\hline$>24$ & 360 & 309 & \\
\hline Smoking, $n$ & & & $p=0.204$ \\
\hline Yes & 15 & 5 & \\
\hline No & 1169 & 745 & \\
\hline Preoperative hypertension, $\mathrm{n}$ & & & $p<0.01$ \\
\hline Yes & 41 & 68 & \\
\hline No & 1143 & 682 & \\
\hline Preoperative hyperlipidemia, $\mathrm{n}$ & & & $p<0.01$ \\
\hline Yes & 382 & 452 & \\
\hline No & 802 & 298 & \\
\hline Tumor location, $\mathrm{n}$ & & & $p=0.651$ \\
\hline Left breast & 619 & 400 & \\
\hline Right breast & 565 & 350 & \\
\hline TNM stage, $\mathrm{n}$ & & & $p=0.388$ \\
\hline Stage I & 417 & 253 & \\
\hline Stage II & 743 & 487 & \\
\hline Stage III & 24 & 10 & \\
\hline Surgical procedures, $\mathrm{n}$ & & & $p<0.01$ \\
\hline Mastectomy & 985 & 713 & \\
\hline Breast conserving operation & 146 & 31 & \\
\hline Breast reconstruction & 53 & 6 & \\
\hline Molecular subtyping, $\mathrm{n}$ & & & $p<0.01$ \\
\hline LuminaA & 73 & 45 & \\
\hline LuminaB & 160 & 173 & \\
\hline Triple negative & 139 & 77 & \\
\hline Her-2 positive & 812 & 455 & \\
\hline
\end{tabular}

$S D$ standard deviation; $B M I$ body mass index

assessed by "West China mood index" scale and their scores were higher than normal. Additionally, all patients examined blood routine examination, blood biochemistry, mammary color ultrasound and breast X-ray before surgery. All patients received general anesthesia and there was no severe adverse reaction after surgery.

\section{Serum lipid alterations after surgery}

The lipid level variations after surgery are shown in Table 2, Figs. 1 and 2. In this study, there were 1151 patients with elevated TG levels, 785 patients with elevated TC levels, 1009 patients with elevated LDL levels and 1548 patients with decreased HDL levels after surgery. For the entire group, we found a significant increase in TG $(p<0.001)$ and LDL $(p<0.001)$, while a marked decrease in HDL $(p<0.001)$ was observed postoperatively. However, in terms of TC, there was no significant difference between pre- and postoperation $(p=0.227)$.

In addition, the participants were then stratified into two groups by preoperative lipid status: preoperative ortholiposis and preoperative hyperlipidemia. For the preoperative ortholiposis group, we found an increase in TG, TC and LDL levels $(p<0.001)$ and a decrease in HDL levels after surgery $(p<0.01)$. For the preoperative hyperlipidemia group, an increase was also noted in the levels of TG and TC between pre- and postoperation $(p<0.001)$, and a decrease was found in both LDL and HDL $(p<0.001)$ after surgery.

Since menopausal status is closely related to serum lipid profiles, the study participants were stratified into two groups by menopausal status: premenopausal and 
Table 2 Comparison of the lipid profiles between pre- and postoperation

\begin{tabular}{|c|c|c|c|}
\hline Parameters & Pre-operation & Post-operation & $p$ \\
\hline \multicolumn{4}{|c|}{ Entire group $(N=1934)$} \\
\hline TG & $1.30 \pm 0.75$ & $1.50 \pm 0.80$ & $<0.001$ \\
\hline $\mathrm{TC}$ & $4.79 \pm 0.91$ & $4.77 \pm 0.96$ & 0.227 \\
\hline $\mathrm{HDL}$ & $1.62 \pm 0.40$ & $1.44 \pm 0.37$ & $<0.001$ \\
\hline LDL & $2.70 \pm 0.74$ & $2.77 \pm 0.77$ & $<0.001$ \\
\hline \multicolumn{4}{|c|}{ Preoperative ortholiposis ( $N=1088$ ) } \\
\hline TG & $0.97 \pm 0.30$ & $1.25 \pm 0.53$ & $<0.001$ \\
\hline $\mathrm{TC}$ & $4.28 \pm 0.55$ & $4.36 \pm 0.72$ & $<0.001$ \\
\hline $\mathrm{HDL}$ & $1.65 \pm 0.34$ & $1.45 \pm 0.33$ & $<0.001$ \\
\hline LDL & $2.30 \pm 0.45$ & $2.45 \pm 0.58$ & $<0.001$ \\
\hline \multicolumn{4}{|c|}{ Preoperative hyperlipidemia $(\mathrm{N}=846)$} \\
\hline TG & $1.72 \pm 0.93$ & $1.82 \pm 0.96$ & $<0.001$ \\
\hline $\mathrm{TC}$ & $5.44 \pm 0.86$ & $5.28 \pm 0.97$ & $<0.001$ \\
\hline $\mathrm{HDL}$ & $1.58 \pm 0.46$ & $1.42 \pm 0.41$ & $<0.001$ \\
\hline LDL & $3.23 \pm 0.71$ & $3.18 \pm 0.79$ & $<0.001$ \\
\hline \multicolumn{4}{|c|}{ Premenopause $(N=1184)$} \\
\hline TG & $1.19 \pm 0.74$ & $1.42 \pm 0.80$ & $<0.001$ \\
\hline $\mathrm{TC}$ & $4.57 \pm 0.83$ & $4.58 \pm 0.90$ & 0.545 \\
\hline $\mathrm{HDL}$ & $1.61 \pm 0.38$ & $1.43 \pm 0.35$ & $<0.001$ \\
\hline LDL & $2.54 \pm 0.67$ & $2.63 \pm 0.72$ & $<0.001$ \\
\hline \multicolumn{4}{|c|}{ Postmenopause $(\mathrm{N}=750)$} \\
\hline $\mathrm{TG}$ & $1.47 \pm 0.75$ & $1.62 \pm 0.80$ & $<0.001$ \\
\hline $\mathrm{TC}$ & $5.13 \pm 0.92$ & $5.06 \pm 0.97$ & 0.007 \\
\hline $\mathrm{HDL}$ & $1.63 \pm 0.43$ & $1.46 \pm 0.39$ & $<0.001$ \\
\hline LDL & $2.97 \pm 0.77$ & $3.00 \pm 0.79$ & 0.186 \\
\hline \multicolumn{4}{|c|}{$\mathrm{BMI} \leq 24(\mathrm{~N}=1265)$} \\
\hline TG & $1.17 \pm 0.59$ & $1.39 \pm 0.67$ & $<0.001$ \\
\hline $\mathrm{TC}$ & $4.73 \pm 0.88$ & $4.72 \pm 0.94$ & 0.573 \\
\hline $\mathrm{HDL}$ & $1.69 \pm 0.40$ & $1.49 \pm 0.37$ & $<0.001$ \\
\hline LDL & $2.63 \pm 0.72$ & $2.71 \pm 0.75$ & $<0.001$ \\
\hline \multicolumn{4}{|c|}{$\mathrm{BMI}>24(\mathrm{~N}=669)$} \\
\hline TG & $1.38 \pm 0.79$ & $1.61 \pm 0.84$ & $<0.001$ \\
\hline TC & $4.67 \pm 0.85$ & $4.69 \pm 0.93$ & 0.338 \\
\hline $\mathrm{HDL}$ & $1.51 \pm 0.33$ & $1.35 \pm 0.33$ & $<0.001$ \\
\hline LDL & $2.67 \pm 0.68$ & $2.74 \pm 0.74$ & $<0.001$ \\
\hline
\end{tabular}

$T G$ triglycerides, $T C$ total cholesterols, $H D L$ high-density lipoprotein, $L D L$ lowdensity lipoprotein, $B M I$ body mass index postmenopausal groups. For the premenopausal group, we found an increase in TG and LDL levels $(p<0.001)$ and a decrease in HDL levels after surgery $(p<0.01)$, while there was no significant difference in TC levels between pre- and postoperation $(p=0.545)$. For the postmenopausal group, an increase was also noted in the levels of TG between pre- and postoperation $(p<0.001)$; a decrease was found in TC $(p=0.007)$ and HDL $(p<0.001)$ after surgery.

Additionally, we stratified the participants into two groups by $\mathrm{BMI}: \mathrm{BMI} \leq 24$ and $\mathrm{BMI}>24$. We found an increase in TG, TC and LDL levels $(\mathrm{p}<0.001)$ and a decrease in HDL levels after surgery $(\mathrm{p}<0.01)$ in both groups.

\section{Incidence of postoperative hyperlipidemia in patients with preoperative ortholiposis}

Among the 1934 patients, 1088 patients had normal serum lipid profiles before surgery. As many patients had increased serum lipid levels after surgery, we further assessed the percentage of patients newly diagnosed with postoperative dyslipidemia (Fig. 3). Among the 1088 patients with preoperative ortholiposis, 302 patients $(27.76 \%)$ were newly diagnosed with dyslipidemia after surgery. Stratified by menopausal status, the incidence of newly diagnosed dyslipidemia was higher in the postmenopausal group (32.31\%) than in the premenopausal $(26.07 \%)$ group $(p=0.041)$. Then, stratified by BMI status, we found that the incidence of newly diagnosed dyslipidemia was higher in patients with BMI $>24$ (34.92\%) than in patients with $\mathrm{BMI} \leq 24 .(24.84 \% ; p=0.001)$.

\section{Comparison of the magnitude of lipid alterations after surgery}

To explore the effects of menopausal status and BMI status on serum lipid level variations, we carried out further analyses. The data is provided in Table 3 and Fig. 4. The levels of TG increased after surgery in both the premenopausal and postmenopausal groups $(p<0.001)$, and the magnitude of the TG increase after surgery in the premenopausal group was greater than that in the postmenopausal group $(p=0.012)$. For $\mathrm{TC}$, the variations in

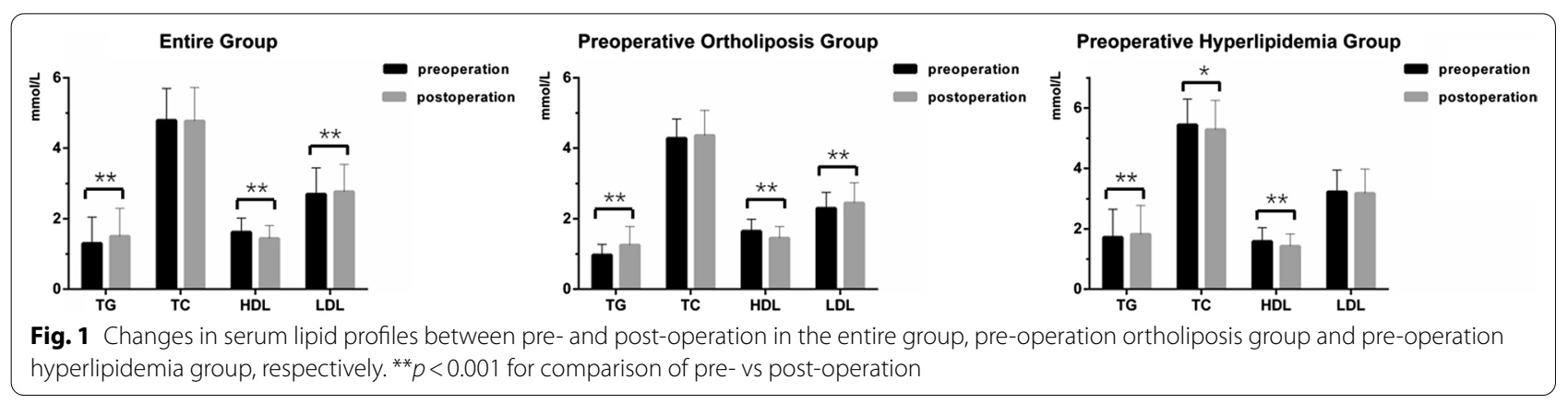




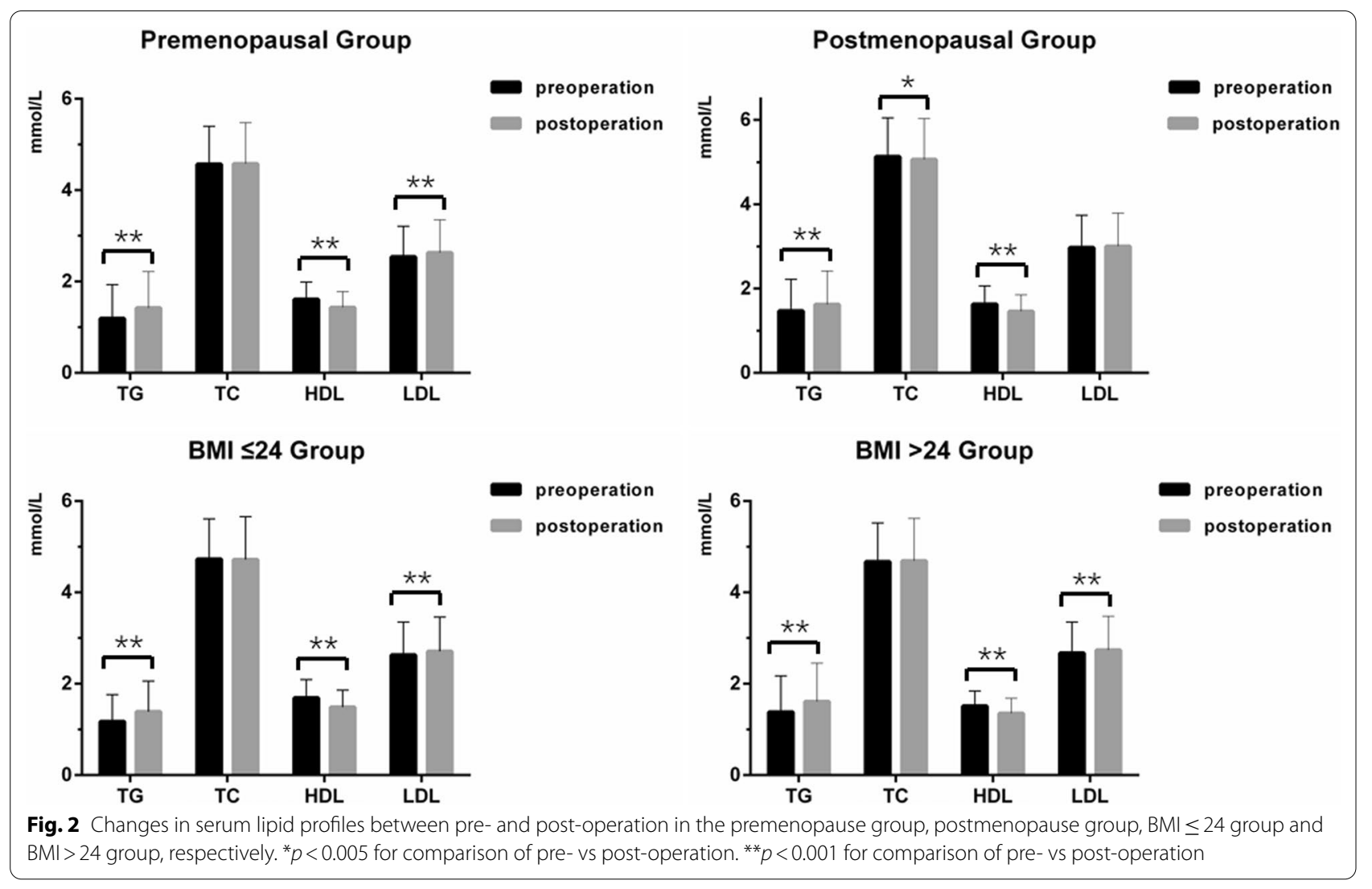

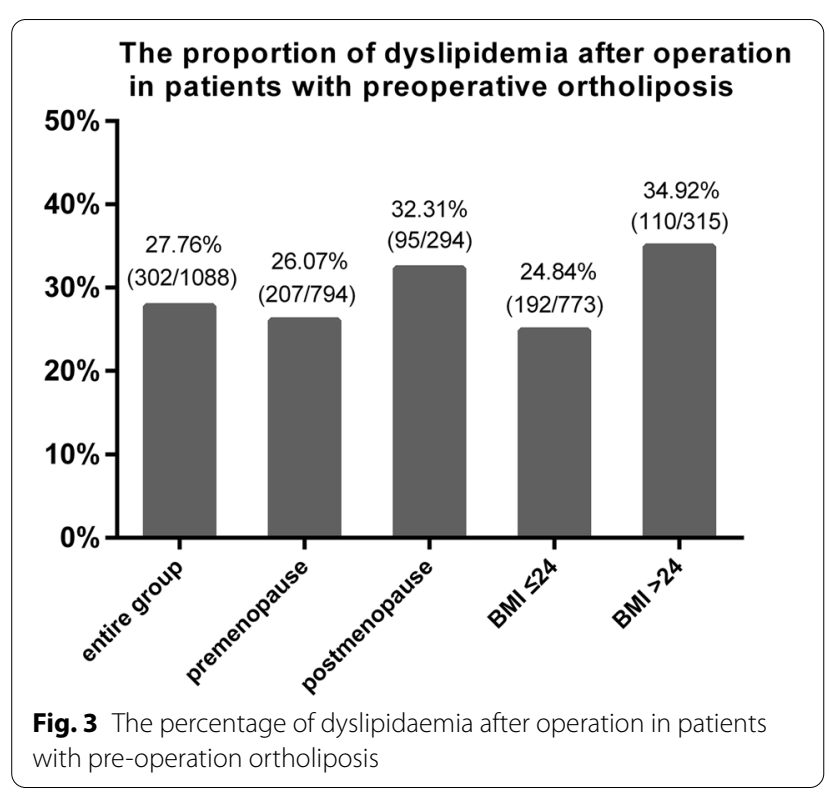

TC pre- and postoperation were significantly different between the premenopausal and postmenopausal groups, and the elevation in TC was greater in the premenopausal group $(p=0.013)$. For HDL, we found a decrease in both the premenopausal and postmenopausal groups $(p<0.001)$, but there was no significant difference in the magnitude of variation in HDL between the groups $(p=0.429)$. For LDL, we found an increase in the premenopausal group $(p<0.001)$, and the magnitude of LDL increase was greater in the premenopausal group than in the postmenopausal group $(p=0.015)$.

Additionally, we compared the magnitude of lipid alterations after surgery between patients with BMI $>24$ and $\mathrm{BMI} \leq 24$. The levels of HDL decreased after surgery in both the $\mathrm{BMI}>24$ and $\mathrm{BMI} \leq 24$ groups, and the magnitude of decreased HDL was greater in the BMI $\leq 24$ group $(p<0.001)$. However, there was no significant difference in the magnitude of variation in TG $(p=0.114)$, $\mathrm{TC}(p=0.481)$ and LDL $(p=0.057)$.

Factors related to the increase in lipid profiles after surgery To explore the potential clinicopathological parameters associated with increased lipid profiles after surgery, we performed univariate and multivariate analyses (Table 4). With respect to TG, TC and LDL levels, multivariate analysis showed that PR-positive status and preoperative hyperlipidemia were associated with elevated TG, TC and LDL levels after surgery $(p<0.05)$. In terms of HDL, both univariate and multivariate analyses showed that 
Table 3 Comparison of change values of blood lipid between pre- and postoperation

\begin{tabular}{lccr}
\hline Parameters & Premenopause $(\mathbf{N}=\mathbf{1 1 8 4})$ & Postmenopause $(\mathbf{N}=\mathbf{7 5 0})$ & $\boldsymbol{p}$ \\
\hline TG & $0.23 \pm 0.69$ & $0.15 \pm 0.63$ & 0.012 \\
TC & $0.013 \pm 0.71$ & $-0.07 \pm 0.72$ & 0.013 \\
HDL & $-0.19 \pm 0.29$ & $-0.18 \pm 0.30$ & 0.429 \\
LDL & $0.09 \pm 0.53$ & $0.03 \pm 0.56$ & 0.015 \\
\hline Parameters & $\mathbf{B M I} \leq \mathbf{2 4}(\mathbf{N}=\mathbf{1 2 6 5 )}$ & $\mathbf{B M I}>\mathbf{2 4}(\mathbf{N}=\mathbf{6 6 9 )}$ & $\mathbf{p}$ \\
\hline TG & $0.22 \pm 0.57$ & $0.17 \pm 0.81$ & $<0.001$ \\
TC & $-0.01 \pm 0.57$ & $-0.04 \pm 0.72$ & 0.689 \\
HDL & $-0.20 \pm 0.30$ & $-0.15 \pm 0.27$ & $<0.001$ \\
LDL & $0.08 \pm 0.53$ & $0.03 \pm 0.58$ & 0.812 \\
\hline
\end{tabular}

$T G$ triglycerides, $T C$ total cholesterols, $H D L$ high-density lipoprotein, $L D L$ low-density lipoprotein

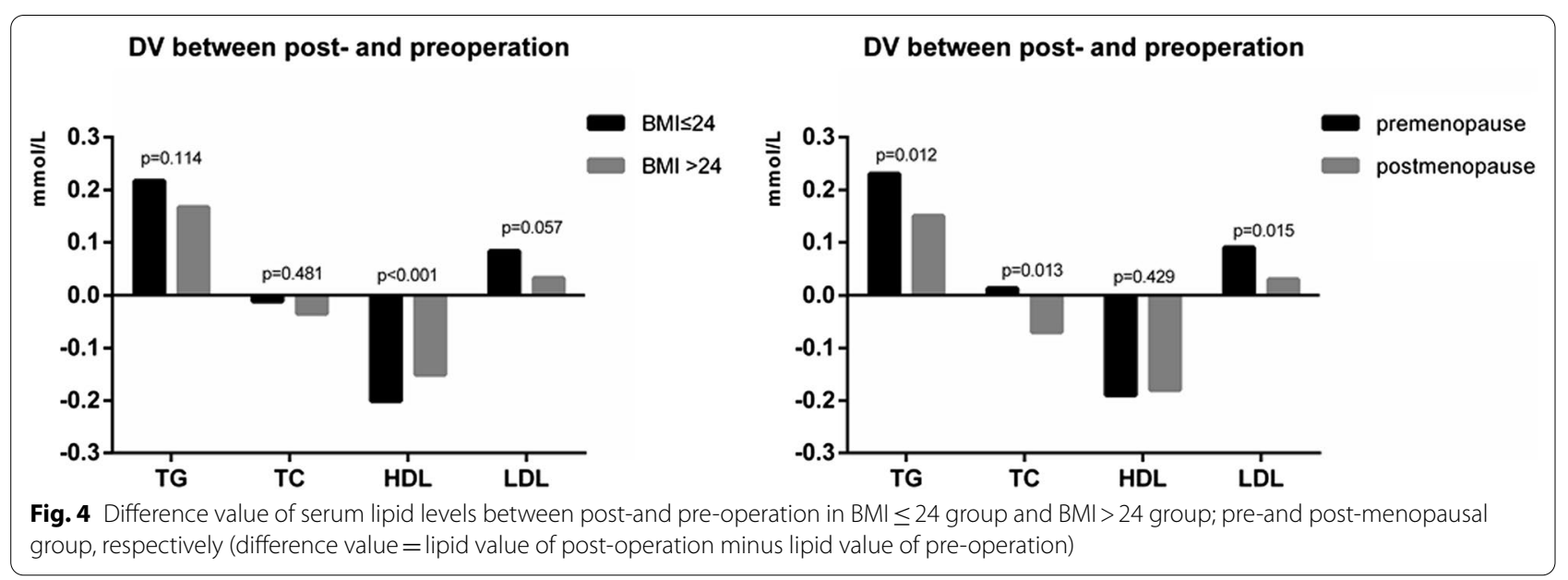

none of the factors were associated with decreased HDL levels after surgery.

\section{Discussion}

In this study, we focused on the variations in serum lipid profiles pre- and postoperation in patients with $\mathrm{BC}$. To our knowledge, this is one of the studies that contains a relatively large sample size to investigate lipid changes after breast cancer surgery. In this entire cohort, we found that serum lipid profiles of BC patients may increase after surgery, especially premenopausal patients. Additionally, postmenopausal and overweight patients may have a higher risk of being diagnosed with dyslipidemia after surgery.

Previously, a few studies have addressed the stress response to trauma and surgery [5]. The activation of the hypothalamic-pituitary-adrenal (HPA) axis and the sympathetic nervous system (SNS) are characteristics of the stress response to surgical trauma [15]. With the activation of the HPA axis and SNS, several hormones, such as adrenocorticotropic hormone (ACTH), catecholamine, glucocorticoids and mineral corticoid, are synthesized and secreted in greater abundance and more rapidly [16]. However, high levels of these hormones would have negative feedback on gonadotropin-releasing hormone (GnRH), and thus, the hypothalamic-pituitary-gonadal axis would be inhibited [17]. In addition, glucocorticoids could directly inhibit the release of estrogen and progesterone secreted by the ovary [18-20]. Notably, the inhibition of gonadotropins and lower estrogen concentrations in the plasma were associated with elevated serum lipid profiles [21]. Furthermore, apart from the activation of the HPA axis, another major metabolic change caused by trauma is a decrease in the normal metabolism of insulin, which means the occurrence of insulin resistance [22-24]. Insulin resistance has been reported to be associated with abnormal lipid metabolism [25]. Additionally, a rise in glucocorticoids has recently been suggested to be involved in insulin resistance and lipid abnormalities [26]. In this study, our findings showed that the serum levels of TG and LDL both increased, and the concentration of HDL decreased after surgical trauma. A possible 
Table 4 Factors identified to be associated with the increase in lipid profiles based on multivariate analysis, by stepwise logistic regression

\begin{tabular}{|c|c|c|c|c|c|c|}
\hline & \multicolumn{3}{|c|}{ Univariate analysis } & \multicolumn{3}{|c|}{ Multivariate analysis } \\
\hline & OR & $95 \% \mathrm{Cl}$ for OR & $\mathbf{p}$ & OR & $95 \% \mathrm{Cl}$ for OR & $\mathrm{p}$ \\
\hline \multicolumn{7}{|c|}{ TG: increase $(n=1151)$ vs not increase $(n=783)$} \\
\hline \multicolumn{7}{|l|}{ Age } \\
\hline$\leq 40$ & 1 & & & & & \\
\hline $40-50$ & 1.067 & $0.825-1.381$ & 0.622 & & & \\
\hline$>50$ & 0.712 & $0.548-0.926$ & 0.011 & & & \\
\hline \multicolumn{7}{|l|}{ Premenopause } \\
\hline Pre- & 1 & & & & & \\
\hline Post- & 0.671 & $0.557-0.808$ & $<0.001$ & & & \\
\hline \multicolumn{7}{|l|}{ ER } \\
\hline ER- & 1 & & & & & \\
\hline ER+ & 1.570 & $1.293-1.905$ & $<0.001$ & & & \\
\hline \multicolumn{7}{|l|}{ PR } \\
\hline PR- & 1 & & & 1 & & \\
\hline $\mathrm{PR}+$ & 1.656 & $1.371-2.000$ & $<0.001$ & 1.411 & $1.046-1.904$ & 0.024 \\
\hline Preoperative hyperlipidemia & 2.212 & $1.838-2.663$ & $<0.001$ & 2.121 & $1.745-2.578$ & $<0.001$ \\
\hline \multicolumn{7}{|c|}{ TC: increase $(n=785)$ vs no increase $(n=1149)$} \\
\hline \multicolumn{7}{|l|}{ Premenopause } \\
\hline Pre- & 1 & & & & & \\
\hline Post- & 0.801 & $0.664-0.966$ & 0.02 & & & \\
\hline \multicolumn{7}{|l|}{ ER } \\
\hline$E R-$ & 1 & & & & & \\
\hline $\mathrm{ER}+$ & 1.253 & $1.029-1.526$ & 0.025 & & & \\
\hline \multicolumn{7}{|l|}{$P R$} \\
\hline PR- & 1 & & & 1 & & \\
\hline $\mathrm{PR}+$ & 1.409 & $1.163-1.707$ & $<0.001$ & 1.479 & $1.093-2.003$ & 0.011 \\
\hline Preoperative hyperlipidemia & 1.710 & $1.419-2.061$ & $<0.001$ & 1.672 & $1.376-2.030$ & $<0.001$ \\
\hline \multicolumn{7}{|c|}{ LDL: increase $(n=1009)$ vs not increase $(n=925)$} \\
\hline \multicolumn{7}{|l|}{ Premenopause } \\
\hline Pre- & 1 & & & & & \\
\hline Post- & 0.813 & $0.676-0.976$ & 0.027 & & & \\
\hline \multicolumn{7}{|l|}{ ER } \\
\hline$E R-$ & 1 & & & & & \\
\hline ER+ & 1.349 & $1.112-1.635$ & 0.002 & & & \\
\hline \multicolumn{7}{|l|}{ PR } \\
\hline$P R-$ & 1 & & & 1 & & \\
\hline $\mathrm{PR}+$ & 1.443 & $1.196-1.740$ & $<0.001$ & 1.407 & $1.164-1.701$ & $<0.001$ \\
\hline Preoperative hyperlipidemia & 1.863 & $1.552-2.237$ & $<0.001$ & 1.838 & $1.530-2.209$ & $<0.001$ \\
\hline
\end{tabular}

$T G$ triglycerides, $T C$ total cholesterols, $L D L$ low-density lipoprotein, $E R$ estrogen receptor, $P R$ progesterone receptor

explanation for the elevated lipid profiles might be that the stress response to surgical trauma, which may lead to the activation of the HPA axis and a rise in glucocorticoids, results in an increase in serum lipid profiles. Additionally, the activation of the HPA axis itself may inhibit the function of the ovary and then decrease estrogen, resulting in higher lipid profiles.
Apart from the neuroendocrine changes caused by the stress response to surgical trauma, surgery itself might also have effects on serum lipid levels. Huang et al. [27] investigated the serum lipid profiles of 600 patients with lung cancer before and after surgical treatment. Their findings showed that the levels of TC and LDL both increased after surgery $(p<0.05)$, and the levels of 
TG and HDL were also increased, although no statistically significant differences were observed. Similarly, our results showed that TG and LDL increased and HDL decreased after surgery. Huang et al. [27] noted that the increase in lipid profiles might be attributed to the fact that the tumor burden was reduced and the functions of organs were improved after surgery; thus, lipid synthesis might increase and consumption might decrease accordingly. As reported, lipids, especially cholesterol, play a vital role in cellular structure and function [28]. Tumor cells grow rapidly and consume many lipids, which results in lower lipid profiles in cancer patients [29-31]. However, the consumption of lipids is reduced to some extent if the tumor is removed; thus, the lipid profiles become elevated. Furthermore, strong evidence has indicated that the levels of TC are negatively correlated with the presence, development or metastasis of breast cancer [32-34]. However, the specific mechanisms between lipid profiles and breast cancer are unclear, so further studies are urgently warranted.

In this study, we found that $27.76 \%$ of patients with preoperative ortholiposis developed dyslipidemia after surgery. Jianxing et al. [35] reported that dyslipidemia was positively correlated with BMI. Similarly, our results also showed that patients with BMI $>24$ had a higher risk of developing dyslipidemia after surgery. In terms of menopausal status, Croce et al. [36] reported that compared with premenopausal women, postmenopausal women had a higher risk of developing dyslipidemia. In accordance with their findings, we also found that postmenopausal patients had a higher risk of developing dyslipidemia than premenopausal patients after surgery.

$\mathrm{Du}$ et al. [37] reported that serum lipid profiles increased significantly after hysterectomy, and the changes were more remarkable in younger women. Similarly, Tian et al. [38] also reported that the younger group showed a greater increase in TC and LDL levels during chemotherapy than the 41-65-year-old group. Consistent with their findings, in our study, it is worth noting that the magnitude of the increased TG, TC and LDL was greater in the premenopausal group than in the postmenopausal group. A possible explanation may be that the neuroendocrine changes caused by the stress response to surgical trauma had more effects on the well-functioning ovaries of premenopausal women [37]. Moreover, it is not difficult to understand that postmenopausal ovarian function is poor; thus, external interference has limited effects on ovarian function.

Furthermore, we found that patients with preoperative dyslipidemia had a higher risk of increased lipid profiles after surgery than those with normal preoperative lipid levels. The reason may be that patients with dyslipidemia may have disorders of lipid metabolism, which makes them more likely to have lipid changes. Additionally, we found that patients with BC with PR positivity had a higher risk of increased lipid profiles after surgery than those with PR negativity. Unfortunately, very few studies have focused on the relationship between PR status and lipid metabolism, and more research should be carried out in the future to confirm this finding.

To the best of our knowledge, the present study was one of the studies with a relatively large sample to provide comprehensive information on the serum lipid profiles pre- and postoperation in both premenopausal and postmenopausal patients with BC. Moreover, factors potentially implicated in elevated lipid profiles after surgery were also identified. However, there are still several limitations that should be highlighted. Notably, the physical activities of the patients were largely reduced, and their diets tended to be high-fat or high-protein during the perioperative period, which might also enhance the elevated lipid profiles. Additionally, there is a lack of data on calorie intake and consumption, energy variation, body composition, axillary surgery procedures, the number of lymph nodes removed and surgery time. The exact mechanism of lipid increase is not yet clear, and many factors may also have intertwining effects on lipids. Last but not the least, as Li et al. [39] reported, preoperative lower TG and HDL-C level were risk factors of breast cancer patients. It is urgent for us to explore the relationship between dyslipidemia and CVD and the long-term prognosis of patients with $\mathrm{BC}$.

\section{Conclusions}

In summary, the serum lipid profiles of patients with $B C$ may increase after surgery, especially in those who are premenopausal. Additionally, quite a few patients developed dyslipidemia after surgery, and postmenopausal and overweight patients may have a higher risk of being diagnosed with dyslipidemia. However, different surgical procedures did not show different effects on lipid profiles. Therefore, lipid monitoring, dyslipidemia prevention and corresponding interventions should be taken into consideration during the perioperative period.

\section{Abbreviations \\ BC: Breast cancer; TG: Triglycerides; TC: Total cholesterols; LDL: Low-density lipoprotein; HDL: High-density lipoprotein; BMI: Body mass index; CVD: Cardio- vascular disease; ECOG: Eastern Cooperative Oncology Group; SD: Standard deviation; ACTH: Adrenocorticotropic hormone; HPA: Hypothalamic-pituitary- adrenal; SNS: Sympathetic nervous system; GnRH: Gonadotropin-releasing hormone; ER: Estrogen receptor; PR: Progesterone receptor; TNM: Tumor Node Metastasis; SPSS: Statistical Package for the Social Sciences.}

\section{Acknowledgements}

The authors gratefully acknowledge the whole staff of the Department of Breast Surgery, West China Hospital, who generously provided assistance in the collection of data throughout the duration of the study. 


\section{Authors' contributions}

JC and QL put forward the concept of this study. TH drafted the preliminary version of this protocol. TH, ZW, YW and XZ contributed to the study search, study selection, data extraction. $\mathrm{XL}, J \mathrm{~L}$ and LD completed the data analysis. JC and QL helped to solve any disagreement and ensured the quality of this study. All authors critically reviewed, revised the final manuscript. All authors read and approved the final manuscript.

\section{Funding}

Sichuan Science and Technology Program (No. 2019YFS0338), National Natural Science Foundation of China (No. 32071284). Funders have had no influence on this work.

\section{Availability of data and materials}

Data are available via direct requests to the corresponding author at West China Hospital and with permission from the Institutional Review Board and Ethics Committee of West China Hospital.

\section{Declarations}

\section{Ethics approval and consent to participate}

Approval for this retrospective analysis was obtained from the Institutional Review Board and Ethics Committee of West China Hospital approved this retrospective study (IRB No. 2016L03115). Data were accessed anonymously, waiving the requirement for consent from study participants. All methods were carried out in accordance with the relevant guidelines and regulations.

\section{Consent for publication}

Not applicable.

\section{Competing interests}

The authors declare no compeing interests.

\section{Author details}

${ }^{1}$ Department of Breast Surgery, West China School of Medicine/West China Hospital, Sichuan University, Chengdu 610041, Sichuan, China. ${ }^{2}$ Laboratory of Molecular Diagnosis of Cancer, West China Hospital, Sichuan University, Chengdu 610041, Sichuan, China. ${ }^{3}$ West China School of Medicine/West China Hospital, Sichuan University, Chengdu 610041, Sichuan, China. ${ }^{4}$ Center of Biostatistics, Design, Measurement and Evaluation (CBDME), Department of Clinical Research Management, West China Hospital, Sichuan University, Chengdu 610041, Sichuan, China. ${ }^{5}$ West China School of Public Health and West China Fourth Hospital, Sichuan University, Chengdu 610041, Sichuan, China. ${ }^{6}$ Chinese Evidence-Based Medicine Center, West China Hospital, Sichuan University, Chengdu 610041, Sichuan, China. ${ }^{7}$ Department of Breast Surgery, West China Hospital, Sichuan University, Chengdu 610041, Sichuan, China.

Received: 4 August 2021 Accepted: 4 November 2021 Published online: 12 November 2021

\section{References}

1. Ganggayah MD, Taib NA, Har YC, Lio P, Dhillon SK. Predicting factors for survival of breast cancer patients using machine learning techniques. BMC Med Inform Decis Mak. 2019;19(1):48.

2. Bray F, Ferlay J, Soerjomataram I, et al. Global cancer statistics 2018: GLOBOCAN estimates of incidence and mortality worldwide for 36 cancers in 185 countries. CA Cancer J Clin. 2018;68(6):394-424.

3. Force USPST, Owens DK, Davidson KW, et al. Medication use to reduce risk of breast cancer: US preventive services task force recommendation statement. JAMA. 2019;322(9):857-67.

4. Medina Velazquez R, Jimenez Diaz L, Fernandez Carrion J, et al. Major ambulatory surgery for the treatment of breast cancer: Factors conditioning conversion to conventional hospitalization. Cir Esp. 2019;97(1):40-5.

5. Desborough JP. The stress response to trauma and surgery. $\mathrm{Br} J$ Anaesth. 2000;85(1):109-17.
6. Krog AH, Thorsby PM, Sahba M, et al. Perioperative humoral stress response to laparoscopic versus open aortobifemoral bypass surgery. Scand J Clin Lab Invest. 2017;77(2):83-92.

7. SimsekT, Simsek HU, Canturk NZ. Response to trauma and metabolic changes: posttraumatic metabolism. Ulus Cerrahi Derg. 2014;30(3):153-9.

8. Beckwitt $\mathrm{CH}$, Clark AM, Ma B, et al. Statins attenuate outgrowth of breast cancer metastases. Br J Cancer. 2018;119(9):1094-105.

9. Gao S, Zhao D, Wang M, et al. Association between circulating oxidized LDL and atherosclerotic cardiovascular disease: a meta-analysis of observational studies. Can J Cardiol. 2017;33(12):1624-32.

10. Mehta LS, Watson KE, Barac A, et al. Cardiovascular disease and breast cancer: where these entities intersect: a scientific statement from the American Heart Association. Circulation. 2018;137(8):e30-66.

11. Gradishar WJ, Anderson BO, Balassanian R, et al. Breast Cancer, Version 4. 2017, NCCN Clinical Practice Guidelines in Oncology. J Natl Compr Cancer Netw. 2018;16(3):310-20.

12. Joint committee issued Chinese guideline for the management of dyslipidemia in adults. 2016 Chinese guideline for the management of dyslipidemia in adults. Zhonghua Xin Xue Guan Bing Za Zhi. 2016:44(10):833-53.

13. Zhi ZFCKZ. 2018 Chinese guideline on menopause management and menopause hormone therapy. Med J Peking Union Med Coll Hosp. 2018;53(11):729-39.

14. Cui Y, Ruan X, Jin J, et al. The pattern of lipids and lipoproteins during the menopausal transition in Chinese women. Climacteric. 2016;19(3):292-8.

15. Zappala G, McDonald PG, Cole SW. Tumor dormancy and the neuroendocrine system: an undisclosed connection? Cancer Metastasis Rev. 2013;32(1-2):189-200.

16. Offner PJ, Moore EE, Ciesla D. The adrenal response after severe trauma. Am J Surg. 2002;184(6):649-53.

17. Lattin CR, Kelly TR. Glucocorticoid negative feedback as a potential mediator of trade-offs between reproduction and survival. Gen Comp Endocrinol. 2019;286:113301.

18. Toufexis D, Rivarola MA, Lara H, Viau V. Stress and the reproductive axis. J Neuroendocrinol. 2014;26(9):573-86.

19. Chrousos GP, Torpy DJ, Gold PW. Interactions between the hypothalamicpituitary-adrenal axis and the female reproductive system: clinical implications. Ann Intern Med. 1998;129(3):229-40.

20. Vermeulen A. Environment, human reproduction, menopause, and andropause. Environ Health Perspect Suppl. 1993;101(2):91-100.

21. Stevenson JC, Chines A, Pan K, Ryan KA, Mirkin S. A pooled analysis of the effects of conjugated estrogens/bazedoxifene on lipid parameters in postmenopausal women from the selective estrogens, menopause, and response to therapy (SMART) trials. J Clin Endocrinol Metab. 2015;100(6):2329-38.

22. Palermo NE, Gianchandani RY, McDonnell ME, Alexanian SM. Stress hyperglycemia during surgery and anesthesia: pathogenesis and clinical implications. Curr Diab Rep. 2016;16(3):33.

23. Peacock TS. Perioperative hyperglycemia: a literature review. AORN J. 2019;109(1):80-6

24. Frisch A, Chandra P, Smiley D, et al. Prevalence and clinical outcome of hyperglycemia in the perioperative period in noncardiac surgery. Diabetes Care. 2010;33(8):1783-8.

25. Nishimura K, Murakami T, Sakurai T, et al. Circulating Apolipoprotein L1 is associated with insulin resistance-induced abnormal lipid metabolism. Sci Rep. 2019;9(1):14869.

26. Priyadarshini E, Anuradha CV. Glucocorticoid antagonism reduces insulin resistance and associated lipid abnormalities in high-fructose-fed mice. Can J Diabetes. 2017;41(1):41-51.

27. Huang A, Pang L, Jiang Y. Changes of serum lipid levels before and after chemotherapy or operation in patients with lung cancer. Labeled Immunoass Clin Med. 2013;20(2):65-8.

28. Weinstein SJ, Wright ME, Lawson KA, et al. Serum and dietary vitamin E in relation to prostate cancer risk. Cancer Epidemiol Biomarkers Prev. 2007;16(6):1253-9.

29. Jafri H, Alsheikh-Ali AA, Karas RH. Baseline and on-treatment high-density lipoprotein cholesterol and the risk of cancer in randomized controlled trials of lipid-altering therapy. J Am Coll Cardiol. 2010;55:2846-54.

30. Umeki S. Decreases in serum cholesterol levels in advanced lung cancer. Respiration. 1993;60:178-81. 
31. Dessi S, Batetta B, Pulisci D. Altered pattern of lipid metabolism in patients with lung cancer. Oncology. 1992;49(6):436-41.

32. Eliassen AH, Colditz GA, Rosner B, Willett WC. Serum lipids, lipidlowering drugs, and the risk of breast cancer. Arch Intern Med. 2005;165(19):2264-71

33. Vatten $\sqcup$, Foss OP. Total serum cholesterol and triglycerides and risk of breast cancer: a prospective study of 24,329 Norwegian women. Cancer Res. 1990;50(8):2341-6.

34. Fiorenza AM, Branchi A, Sommariva D. Serum lipoprotein profile in patients with cancer, A comparison with non-cancer subjects. Int J Clin Lab Res. 2000;30(3):141-5.

35. Yu J, Tao Y, Dou J, et al. The dose-response analysis between BMI and common chronic diseases in northeast China. Sci Rep. 2018;8(1):4228.

36. Di Croce L, Bruscalupi G, Trentalance A. Independent behavior of rat liver LDL receptor and HMGCoA reductase under estrogen treatment. Biochem Biophys Res Commun. 1996;224(2):345-50.
37. Li D, Xin Z. Changes of endocrine and serum lipid metabolism in women after hysterectomy. J Bengbu Med Coll. 2003;28(2):124-7.

38. Tian W, Yao Y, Fan G, et al. Changes in lipid profiles during and after (neo) adjuvant chemotherapy in women with early-stage breast cancer: a retrospective study. PLoS ONE. 2019;14(8):e0221866.

39. Li $\mathrm{X}$, Tang $\mathrm{H}$, Wang J, et al. The effect of preoperative serum triglycerides and high-density lipoprotein-cholesterol levels on the prognosis of breast cancer. Breast. 2017;32:1-6.

\section{Publisher's Note}

Springer Nature remains neutral with regard to jurisdictional claims in published maps and institutional affiliations.
Ready to submit your research? Choose BMC and benefit from:

- fast, convenient online submission

- thorough peer review by experienced researchers in your field

- rapid publication on acceptance

- support for research data, including large and complex data types

- gold Open Access which fosters wider collaboration and increased citations

- maximum visibility for your research: over $100 \mathrm{M}$ website views per year

At BMC, research is always in progress.

Learn more biomedcentral.com/submissions 